\title{
A scaleless wings mutant associated with tracheal system developmental deficiency in wing discs in the silkworm, Bombyx mori
}

\author{
QINGXIANG ZHOU ${ }^{1,2}$, SHUNMING TANG ${ }^{2}$, YIN CHEN ${ }^{2}$, YONGZHU YI ${ }^{2}$, ZHIFANG ZHANG*,1,2 \\ and GUIFANG SHEN ${ }^{1}$ \\ ${ }^{1}$ Biotechnology Research Institute, Chinese Academy of Agricultural Sciences, Beijing, China and ${ }^{2}$ The Sericultural Research Institute, \\ Chinese Academy of Agricultural Sciences, Zhenjiang City, Jiangsu Province, China
}

\begin{abstract}
A mutant of Bombyx mori has wings with few scales and is named scaleless. We investigated the morphology of this mutant and found that it had many fewer wing scales than the corresponding wild type (WT) silkworm and that the remaining scales were smaller in shape with fewer furcations. Reciprocal transplantation of wing discs between scaleless and WT revealed that the WT wing disc could develop into a small wing with scales after transplantation into a scaleless larva; however, the scaleless wing disc developed into a small wing without scales in a WT larva. Upon dissection of WT and scaleless wing discs at different stages from the fifth instar larva to adulthood, no obvious differences were found before pupation. However, after pupation, tracheae produced from WT wing veins extended to the lacunae between the veins and formed a network on the second day after pupation, whereas this did not happen in scaleless. At the same time, no marked difference in adult body tracheal development was found between the mutant and wild type. Furthermore, if the surface of a wing disc was cut and its veins injured, the resulting wing also had fewer scales than the corresponding WT. Also, we found that higher partial pressure of $\mathrm{O}_{2}$ could rescue the loss of scales in scaleless. These data suggest that the factors affecting the growth of scales were not produced in the hemolymph, but in the wing disc itself. It is also implied that wing scale development is dependent on the correct organization of the tracheal system in the wing disc.
\end{abstract}

KEY WORDS: Bombyx mori, scaleless, scale, transplantation, trachea

\section{Introduction}

The wing disc is one of the larval primordia of holometabolous insects and gives rise to the wing of the adult. The wing disc grows slowly during the larval period and develops rapidly after the wandering stage. It is also reported that the development of the wing disc is dependent on hormones and other factors in the hemolymph (Kawasaki and Iwashita, 1987; Kango-Singh et al., 2001). Wing mutants are important for studying the development of the insect wing disc or wing. In the silkworm, Bombyx mori, there are three "wingless" mutants: $f$, rwand $V g$. The recessive f/mutant has the most extreme phenotype among the three, which leads to undetectable wings at the pupal and adult stages. The morphology of the four wing discs in the homozygous f/larva is normal at least until the fourth instar. But after the last larval ecdysis, no tracheae can be found migrating into the lacunal spaces in the $f /$ wing discs. This causes the $f /$ wing discs to atrophy
(Fujiwara and Hojyo, 1997). Using reciprocal transplantations of wing discs, Hojyo and Fujiwara (1997) demonstrated that the factors controlled the $\mathrm{f} /$ mutant did not come from the hemolymph, but from the wing discs.

The coating of scales on the surface of the wings is a basic characteristic of lepidopteran adults. Patterns formed by multicolored scales are not only for mimicry, but also a key innovation during lepidopteran evolution. Consequently, the understanding of the formation of butterfly wing patterns is important for the study of lepidopteran evolution and taxonomy (Galant et al., 1998). Butterfly wing patterns are primarily the result of the deposition of colored pigments, which is gradually completed during wing scale

Abbreviations used in this paper: ASC, achaete-scute complex; ASH, ASC homolog; fl, flügellos; nlw, non lepies wings; rw, rudimentary wing; Vg, Vestigial; WT, wild type.

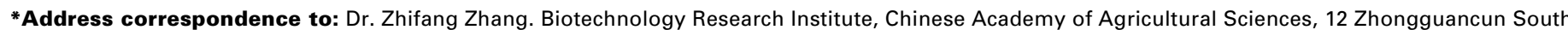
Street, Beijing 100081, China. Fax: +86-10-6897-5136. e-mail: zhifangzhang@yahoo.com
} 

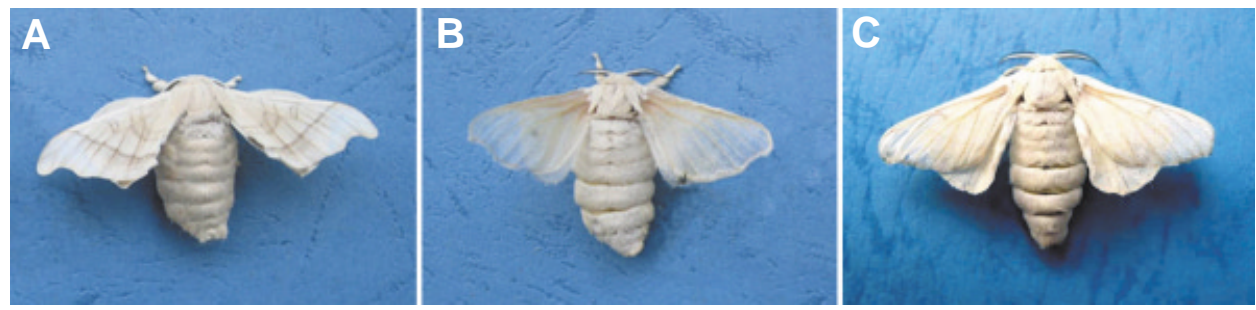

Fig. 1. Silkmoths of Wild type (A), scaleless under atmospheric conditions (B) and scaleless under $\mathbf{5 0} \% \mathbf{O}_{\mathbf{2}}(\mathbf{C})$. There are obviously many fewer wing scales in scaleless (B) than in WT (A) and the wings of scaleless seem to be transparent. There are many more wing scales in scaleless under $50 \% \mathrm{O}_{2}(\mathrm{C})$ than under normal atmospheric conditions.
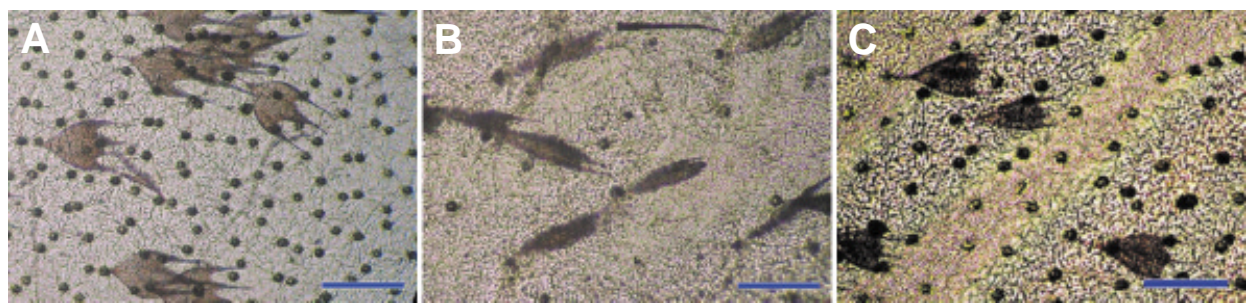

Fig. 2. Enlarged portion of the wings. (A) Most scales of WT were washed off before observation. Sockets (black dots) are arranged compactly, while the wings of scaleless (B) appear in their natural state, without washing. Few sockets are present. It also can be seen that scales in scaleless are not only fewer but smaller than in WT and that not all of the sockets support scales. (C) It is obvious that socket cells in moths developed under $50 \% \mathrm{O}_{2}$ are much more abundant than that under normal atmospheric conditions. Most of the scales in (C) were washed off before observation. Bar, $100 \mu \mathrm{m}$.

determine whether or not the critical factors responsible for the scaleless mutant were produced in the hemolymph. We also tried to break the tracheal system in the WT wing disc and to maintain the scaleless pupae under higher partial pressure of $\mathrm{O}_{2}$ in order to study the effects of respiration on scale development.

\section{Results}

Morphological comparison between WT and scaleless moth wings

There were no obvious differences in appearance between the WT and scaleless silkworms at larval and pupal stages. However, after eclosion, distinct differences were apparent between the two strains. The wing surface of WT moths was fully covered with scales (Fig. 1A), whereas the scaleless wings were covered only by a few scales with transparent looking (Fig. 1B). After washing off most of the scales, many sockets, through which scales are secreted, were visible in the WT wings under the

maturation (Koch et al., 2000a; Koch et al., 2000b). Studies have also showed that the formation of some wing patterns is controlled by nested expressions of regulatory genes (Brunetti et al., 2001).

The Drosophila achaete-scutecomplex ( $A S C$ ), a cluster of four proneural genes, plays an indispensable role in the development of bristles, a homologous structure of lepidopteran wing scales. $A S H 1$, a homolog of the Drosophila ASC genes, has been cloned from the butterfly Precis coenia. It has been revealed that $A S H 1$ can promote the initial events of selection and formation of both neural and scale precursor cells. It was also suggested that tracheal migration is necessary for the expression of $\mathrm{ASH} 1$ (Galant et al., 1998). The wing vein is one of the main parts of the insect wing. It not only provides structural support to the wing, but also carries tracheae and nerves (Garcia-Bellido and de Celis, 1992; Roch et al., 1998). Tracheae consist of a large tubular network that provides air to virtually every cell in the organism. Through the spiracles, oxygen enters into this network, diffuses along the major tracheae and finally reaches the tracheoles, which end blindly on the surface of tissues (Samakovlis et al., 1996; Ebner et al., 2002).

We maintain a silkworm mutant with scaleless wings, which was collected from the countryside of Hubei Province, China in the early 1990's. The wings of the mutant moth are obviously covered with fewer scales than the wild type (WT) and appear transparent. It has been shown by genetic analysis that the morphological character of this mutant is controlled by a recessive gene, $n / w$ (non lepies wings, which we call here scaleless, for a more accurate term). The vitality of scaleless is no worse than that of WT (Zhang and Qin, 1991). In this work, we investigated the major differences between the wing discs and the wings of WT and scaleless. We used a reciprocal transplantation assay to microscope (Fig. 2A). In contrast, only a few sockets were present in the wings of scaleless moths and not all of the sockets supported scales (Fig. 2B). We also found fewer furcations of each scale on the scaleless wings than on WT wings. These data demonstrated clear, characteristic morphological differences between scaleless and WT wings.

\section{Reciprocal transplantation of wing discs between WT and scaleless}

We dissected out wing discs from 50 WT or scaleless one-day old fifth instar larvae and transplanted WT wing discs to scaleless larvae or scaleless wing discs to WT larvae. About half of the acceptor silkworms developed to moths, each with an additional small wing on the back between the $4^{\text {th }}$ and $5^{\text {th }}$ abdominal arthromeres. Eighteen out of the 28 (about 64\%) transplanted WT wings that developed in scaleless moths were covered with scales (Fig. 3A), but none of the 23 scaleless transplanted wings into WT

TABLE 1

\section{NUMBER OF SOCKET CELLS}

\begin{tabular}{lcccc} 
Sort & Wing number & $\begin{array}{l}\text { Mean socket } \\
\text { cell number } \\
\text { per } \mathbf{~ m}^{2}\end{array}$ & Standard deviation & T grouping \\
\hline WT & 10 & 499 & 12 & $\mathrm{~A}$ \\
scaleless & 10 & 51 & 8 & $\mathrm{~B}$ \\
scaleless $+50 \%$ O2 & 10 & 376 & 70 & $\mathrm{C}$ \\
\hline
\end{tabular}

WT stands for wild type, scaleless stands for the scaleless wing mutant, and scaleless $+50 \% \mathrm{O} 2$ stands for scaleless developed under $50 \% \mathrm{O}_{2}$ partial pressure. Cell numbers per $\mathrm{mm}^{2}$ wing portion of the three sorts are significantly different. While the standard deviation of scaleless + $50 \% \mathrm{O}_{2}$ is much larger than that of the others. Different letters in the column named "T grouping" indicate a statistically significant difference (ANOVA, $p<0.01$ ). 

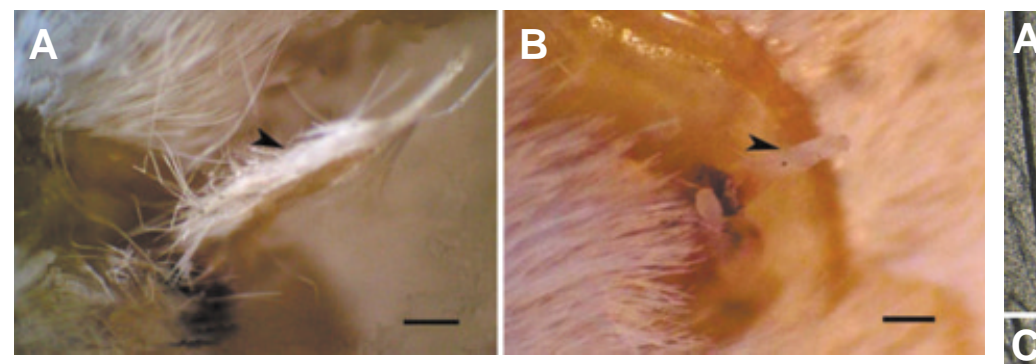

Fig. 3 (Above). Small wings developed from transplanted wing discs. Note that (A) the wing developed from a transplanted WT wing disc into a scaleless silkmoth is covered with scales, while (B) no scales are present on the wing developed from a transplanted scaleless wing disc into a WT silkmoth. Arrows show the transplanted wings. Bar, $1 \mathrm{~mm}$.

Fig. 4 (Right). Differences between pupal wings of scaleless and WT. On the
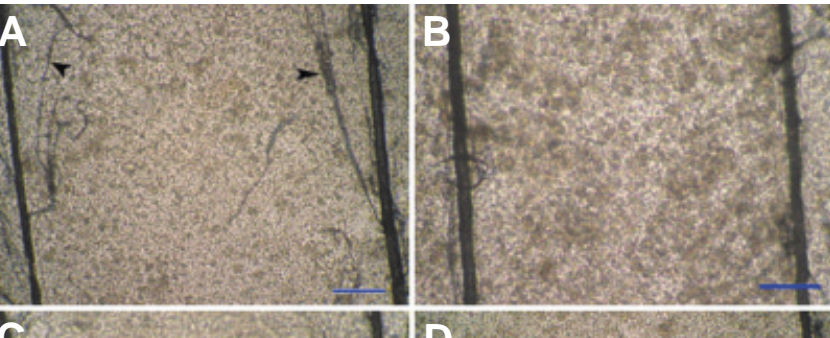

first pupal day (A), many tracheae protrude from the vein and have invaded into wing lacuna between the veins in WT (arrows), while (B) there are almost no tracheae in the wing lacuna between the veins in scaleless. On the second pupal day (C), more tracheae have invaded into the wing lacuna between the veins than in the 1 d pupa in WT (arrows). (D) There are still no tracheae in the wing lacuna between the veins in scaleless or only tracheal cluster buds just protrude out of the veins (arrows). Bar, $100 \mu \mathrm{m}$.

moths had any scales (Fig. 3B). This suggested that the scaleless phenotype might be controlled by regulatory factors acting within the wing discs and not by diffusible hemolymph factors.

Upon examining the microstructure of the small wings, we found that they contained veins. Moreover, many tracheae emanated from the acceptor or the transplanted wing disc and linked the transplanted wing disc to its acceptor. These observations suggested that the small wings had developed from the transplanted wing discs and that tracheae played an important role in their growth.

\section{Dissection and observation of WT and scaleless 5th instar larval wing discs, pupal wings and the adult body tracheae}

Since the factors controlling the scaleless phenotype were found to be acting within the wing discs, we focused our attention on the structure of the wing discs of scaleless. In order to define the developmental time responsible for the scaleless wing defect and determine the major differences between the microstructure of WT and scaleless wings, we compared the morphology of the $5^{\text {th }}$ instar larval wing discs and pupal wings. There seemed to be no morphological differences until the onset of pupation. After pupation, however, tracheal clusters began to develop from the veins and tracheoles protruding from tracheal clusters could be seen in the wings of day 1 WT pupae but not found in scaleless (Fig. 4 A,B). The divergence was more obvious in day 2 pupae (Fig. 4 C,D), when more tracheoles spread from tracheal clusters of WT wings, extending to the lacunae between the veins and forming a network. By contrast, there was almost no outgrowth of tracheae in the scaleless wing. The differences remained similar until the day 6 pupal stage, when WT wings were covered with scales and tracheoles could no longer be seen clearly.

We observed the tracheal system of the adult body carefully and found that there were no obviously differences between the scaleless and WT.

\section{Effects of injuring the wing disc surface on wing and scale development}

Making cuts on the surface of early WT fifth instar larval wing discs resulted in a decrease in the number and size of scales on the adult wing (Fig. 5B). There were also fewer wing sockets than in uninjured wings and not all of the sockets supported scales. The forewing that developed from the injured forewing disc was smaller than the controls' and almost as small as the hindwing on the same side (Fig. 5A). Fifteen of the twenty treated larvae metamorphosed into moths; of these, the results from two moths were in accord with the above results in our experiments, eleven developed into moths without right forewings, while the remainder developed normally.

\section{Effects of higher partial pressure of $\mathrm{O}_{2}$ on the development of wing scales}

The developmental duration of the pupae maintained under $50 \% \mathrm{O}_{2}$ partial pressure was about one day longer than that under normal atmospheric pressure and the wing surface of the scaleless moths under $50 \% \mathrm{O}_{2}$ were covered with much more scales than that under normal condition. Socket cells between cubitus 1 and cubitus2 were counted and analyzed. The results showed that the socket cells of moths under $50 \% \mathrm{O}_{2}$ were significantly more than those of moths under normal conditions, though the standard deviation of the cell number was greater (Figs. 1C,2C and Table 1).

It was a regret that pupae raised under $90 \% \mathrm{O}_{2}$ all died due to reasons were unable to determine.

\section{Discussion}

The results from the present study showed that the scalelessmoth carried many fewer wing scales than the WT did (Fig. 1 A,B and Fig. $2 A, B)$. We assume that the genes controlling the formation of scales may be mutated. The covering of wings with scales is a basic character of the Lepidoptera, which distinguishes them from other insect species. It has been proposed that development of dipteran bristles and lepidopteran wing scales occur in a similar manner; the $A S C$ homolog, $A S H 1$, a multifunction factor, also promoted the selection and forming of scale precursor cells (Galant et al., 1998). Studies have shown that the loss of function mutations in the achaete-scute complex ( $A S C$ ) in Drosophila, which includes some proneural genes, can cause a loss of bristles (Cubadda et al., 1997; 
Long et al., 2000; Culi et al., 2001). Moreover, molecular variation at several well-characterized loci involved in neural development has proven to be associated with naturally occurring quantitative variation in bristle number (Long et al., 1998; Long et al., 2000; Robin et al., 2002; Norga et al., 2003). Therefore we believe that a study of the scaleless mutant could help with understanding the development of wing scales in the Lepidoptera and perhaps even sensory bristles in Drosophila.

Our first step in analyzing the regulatory mechanism underlying the scaleless mutation was carried out reciprocal transplants of $5^{\text {th }}$ larval instar wing discs between WT and scaleless. Wing discs undergo complete differentiation in response to ecdysone. We chose one-day old fifth instar larvae for transplantation in this experiment because studies had shown that it was too late for acceptance of the transplanted wing discs after day 4 of the fifth instar (Hojyo and Fujiwara, 1997). It should be noted that transplanted wings could not form in the acceptor moths when we placed the naked wing discs into the open spaces of the abdominal segments. Most of these wing discs were encapsulated and oxidized to nigrescence, or disappeared from the transplanted sites. These results were consistent with those of Hojyo and Fujiwara (1997). In order to avoid this problem, we transplanted the wing disc together with some of its major tracheae just under the epidermis. Under these conditions, the wing disc was able to grow into a small wing on the abdomen of the acceptor moth. Sixty-four percent of the WT wings developed on scaleless acceptor moths had a scale distribution, but none of the scaleless wings that developed from WT acceptor moths were covered with any scales (Fig. 3). The data showed that the regulatory factors controlling the scaleless wing morphology were not present in the hemolymph or endocrine system, but were more likely produced within the wing discs or tissues sticking to them.

There were many tracheae connecting the small wing with the tissues of the acceptor, but only a few, even no tracheae connecting the transplanted wing discs that failing to develop into wings. This showed that the development of silkworm wings might be dependent on the tracheal system. Oxygen has been shown to play a key role not only in the development of wing discs, but also in the formation of scales. Wing discs cultured in vitro grow faster under higher $\mathrm{O}_{2}$ partial pressure and scales form four days earlier under $95 \% \mathrm{O}_{2}+5 \% \mathrm{CO}_{2}$ than undernormal atmospheric conditions (Kawasaki and Iwashita, 1987). Furthermore, the defect underlying the Bombyx wingless mutant, $f$, affects the failure of tracheal migration in wing lacunae (Fujiwara and Hojyo, 1997). Because of these results, we gave attention to the structure of wing tracheae.

In our anatomic experiment, no obvious differences were observed between wing discs of scaleless and those of WT before spinning and even up to pupation. The most obvious distinctions occurred from day 2 pupae onward, when tracheae extended from the WT wing veins and spread into the wing lacunae between the veins, forming a network. On the contrary, almost no tracheae protruded from the scalelesswing veins and no tracheal network was formed (Fig. 4). The tracheae comprise the main part of the insect respiratory system, which delivers oxygen directly to the tissues (Samakovlis et al., 1996). So the failure of scaleless wing tracheal migration to complete the tracheal network, resulting in insufficient oxygen supply, might prevent the differentiation of socket and scale cells.

Since the vitality of scaleless is no worse than that of WT (Zhang and Qin, 1991), are there developmental differences between the body tracheal system of scaleless and WT? In order to clarify this
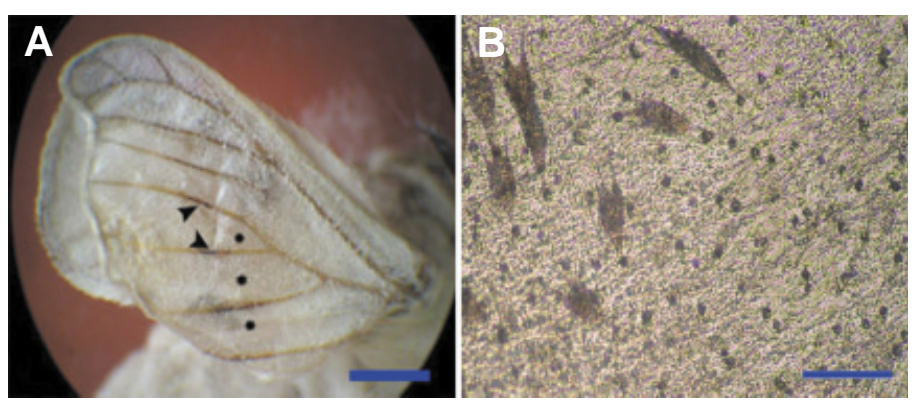

Fig. 5. Adult forewing from a forewing disc that was injured at early $\mathbf{5}^{\text {th }}$ instar. (A) Note that the size of the forewing, which should be larger than the hindwing, was about equal to that of the hindwing and that some areas of this forewing have only a few scales (asterisks). The arrows indicate a crease produced by cutting the wing disc and the blackened portion of the vein. (B) An enlarged portion of the wing. This preparation was made without washing. The sockets and scales are fewer than in WT (Fig. 2A) but more than in scaleless (Fig. 2B). Note that not all of the sockets support scales. Bars, $3 \mathrm{~mm}$ in (A) and $100 \mu \mathrm{m}$ in (B).

question, we anatomized and observed the tracheal system of the adult body. The result was that there was no body tracheal reduction of the mutant compared with WT, this gave further evidence towards our previous conclusion that it was wing disc-specific factors' mutation caused the mutated phenotype of scaleless. Though the yield in the artificial injury experiment was low, for the putrescence of the injured wing discs caused by a possible too deep cut or intactness of the targeted wing discs caused by a possible too shallow cut, it is notable that we were able to mimic the phenotype of few wing scales by making a shallow cut on the surface of the wing disc. Parts of the wing veins that developed from the injured wing disc were darkened (Fig. 5). It is well known that tracheae and nerves in the wing are scabbarded in the veins (Garcia-Bellido and de Celis, 1992). The results reported here are consistent with the hypothesis that the wounding of the tracheae and nerves might disturb the circulation of oxygen in the wing caused oxygen insufficiency, or the disturbance of signaling between tracheae and surrounding tissues, resulting in the reduction of the wing scales. Higher partial pressure of $\mathrm{O}_{2}$ could rescue the loss of scales of the scaleless mutant (Figs. $1 \mathrm{C}, 2 \mathrm{C}$ and Table 1). This strengthened our point that the few of tracheae in the wing, resulting in insufficient oxygen supply, might be the causation of the failure of scale development in scaleless.

In conclusion, the development of scales in the Lepidoptera might depend on a well-organized tracheal system in the wings, which could supply enough oxygen for the development of the scale precursor cells. It is well known that there are some pathways of the origination, migration and formation of tracheal system and there are also ways of scale development and wing pattern formation. Our results show that scale development may be related to tracheal migration. We think there must be some way controlling this connection and it will be an interesting way to further the study of the development of lepidopteran wing scales, also possibly dipteran bristles.

\section{Materials and Methods}

\section{Animals}

The wild type (JY-I) and the scaleless mutant (Shennong-No.1) silkworm stocks are both maintained in our laboratory. The first through fourth instar larvae were reared on mulberry leaves under standard 
conditions (Lü 1990). The newly molted $5^{\text {th }}$ instar larvae were segregated immediately and maintained at $25^{\circ} \mathrm{C}$ with $70 \%-80 \%$ relative humidity for the experiments.

\section{Comparison of the morphology of wings and wing discs between WT and scaleless silkworms}

Wings of WT moths were cut off with surgical scissors and most scales covering the wings were washed off using PBS buffer $[137 \mathrm{mmol} / \mathrm{L} \mathrm{NaCl}$, $2.7 \mathrm{mmol} / \mathrm{L} \mathrm{KCl}, 10 \mathrm{mmol} / \mathrm{L} \mathrm{Na}_{2} \mathrm{HPO}_{4}$ and $\left.2 \mathrm{mmol} / \mathrm{L} \mathrm{KH}_{2} \mathrm{PO}_{4}\right]$. After they dried, the wings were observed under a microscope and photographed. The scaleless wings, which were covered with few scales, were observed without washing. Larval wing discs and pupal wings were dissected with a scalpel and forceps. After rinsing in PBS buffer, differences in wing morphology between WT and scaleless were recorded. Tracheal development in the adult body of scaleless and WT was also carefully compared.

\section{Transplantation of wing discs}

One-day old (day 1) donor and acceptor fifth instar larvae were disinfected with $70 \%$ ethanol and anesthetized in sterile ice water. The dorsal membrane between the fourth and the fifth abdominal segments of the acceptor was cut with a scalpel. Then the right forewing disc of the donor was dissected out and immediately placed into the body of the acceptor through the cut opening using forceps. The whole operation was undertaken on ice and completed within 3 minutes. Operated acceptors were maintained at $4^{\circ} \mathrm{C}$ for about 1 hour, then placed under normal conditions and fed. Each kind of transplantation, WT to scaleless and scaleless to WT, was repeated on more than 50 silkworms.

\section{Artificial injury on the surface of wing discs}

After disinfection with $70 \%$ ethanol and anesthetization in sterile ice water, a shallow transverse cut was made with a scalpel on the surface of the right forewing disc of a day 1 fifth instar WT larva. After maintaining it at $4^{\circ}$ for 1 hour, the larva was placed under normal conditions and fed until metamorphosis. The wings of the injured and those of the control adults were observed under a dissecting microscope. Twenty silkworms were used for this experiment.

\section{Rising pupae under higher partial pressure of $\mathrm{O}_{2}$}

One-day old pupae of scaleless were maintained at higher partial pressure of $\mathrm{O}_{2}, 50 \%$ of $\mathrm{O}_{2}$ or $90 \%$ of $\mathrm{O}_{2}$, until eclosion. Then the wings of the adults were observed and the socket cells of the scales were counted.

\section{Acknowledgments}

We thank Prof. Marian R. Goldsmith at University of Rhode Island for the helpful discussion and critical reviews. This work was supported by grants from the National Natural Science Foundation of China (No: 30271007).

\section{References}

BRUNETTI, C. R., SELEGUE, J. E., MONTEIRO, A., FRENCH, V., BRAKEFIELD, P. M. and CARROLL, S. B. (2001). The generation and diversification of butterfly eyespot color patterns. Curr. Biol. 11: 1578-1585.

CUBADDA, Y., HEITZLER, P., RAY, R. P., BOUROUIS, M., RAMAIN, P., GELBART, W., SIMPSON, P. and HAENLIN, M. (1997). U-shaped encodes a zinc finger protein that regulates the proneural genes achaete and scute during the formation of bristles in Drosophila. Genes Dev. 11: 3083-3095.
CULI, J., MARTIN-BLANCO, E. and MODOLELL, J. (2001). The EGF receptor and $\mathrm{N}$ signalling pathways act antagonistically in Drosophila mesothorax bristle patterning. Development 128: 299-308.

EBNER, A., KIEFER, F. N., RIBEIRO, C., PETIT, V., NUSSBAUMER, U. and AFFOLTER, M. (2002). Tracheal development in Drosophila melanogasteras a model system for studying the development of a branched organ. Gene 287: 55-66.

FUJIWARA, H. and HOJYO, T. (1997). Developmental profile of wing imaginal discs of flügellos (f), a wingless mutant of the silkworm, Bombyx mori. Dev. Genes Evol.207: 12-18.

GALANT, R., SKEATH, J. B., PADDOCK, S., LEWIS, D. L. and CARROLL, S. B. (1998). Expression pattern of a butterfly achaete-scute homolog reveals the homology of butterfly wing scales and insect sensory bristles. Curr. Biol. 8: 807-813.

GARCIA-BELLIDO, A. and DE CELIS, J. F. (1992). Developmental genetics of the venation pattern of Drosophila. Annu. Rev. Genet. 26: 277-304.

HOJYO, T. and FUJIWARA, H. (1997). Reciprocal transplantation of wing discs between a wing deficient mutant ( $f$ ) and wild type of the silkworm, bombyx mori. Dev. Growth Differ. 39: 599-606.

KANGO-SINGH, M., SINGH, A. and GOPINATHAN, K. P. (2001). The wings of Bombyx moridevelop from larval discs exhibiting an early differentiated state: a preliminary report. J. Biosci. 26: 167-177.

KAWASAKI, H. and IWASHITA, Y. (1987). Differentiation of wing disc of the fifth larval instar of Bombyx morï in vitro culture condition. J. Seric. Sci. Jpn. 56: 65-71.

$\mathrm{KOCH}$, P. B., BEHNECKE, B. and FFRENCH-CONSTANT, R. H. (2000a). The molecular basis of melanism and mimicry in a swallowtail butterfly. Curr. Biol. 10 591-594.

KOCH, P. B., LORENZ, U., BRAKEFIELD P. M. and FFRENCH-CONSTANT, R. H. (2000b). Butterfly wing pattern mutants: developmental heterochrony and coordinately regulated phenotypes. Dev. Genes Evol. 210: 536-544.

LONG, A. D., LYMAN, R. F., LANGLEY, C. H. and MACKAY, T. F. (1998). Tow sites in the Deltagene region contribute to naturally occurring variation in bristle number in Drosophila melanogaster. Genetics 149: 999-1017.

LONG, A. D., LYMAN, R. F., MORGAN, A. H., LANGLEY, C. H. and MACKAY, T. F. (2000). Both naturally occurring insertions of transposable elements and intermediate frequency polymorphisms at the achaete-scute complex are associated with variation in bristle number in Drosophila melanogaster. Genetics 154: 1255-1269.

LÜ, H. S. (1990). The sericultural science in China. Shanghai scientific and technical publishers, Shanghai.

NORGA, K. K., GURGANUS, M. C., DILDA, C. L., YAMAMOTO, A., LYMAN, R. F. PATEL, P. H., RUBIN, G. M., HOSKINS, R. A., MACKAY, T. F. and BELLEN, H. J. (2003). Quantitative analysis of bristle number in Drosophila mutants identifies genes involved in neural development. Curr. Biol. 13: 1388-1396.

ROBIN, C., LYMAN, R. F., LONG, A. D., LANGLEY, C. H. and MACKAY, T. F. (2002) Hairy: A quantitative trait locus for Drosophilasensory bristle number. Genetics 162 155-164.

ROCH, F., BAONZA, A., MARTIN-BLANCO, E. and GARCIA-BELLIDO, A. (1998). Genetic interactions and cell behaviour in blisteredmutants during proliferation and differentiation of the Drosophilawing. Development 125: 1823-1832.

SAMAKOVLIS, C. HACOHEN, N., MANNING, G., SUTHERLAND, D. C. GUILLEMIN, K. and KRASNOW, M. A. (1996). Development of the Drosophilatracheal system occurs by a series of morphologically distinct but genetically coupled branching events. Development 122: 1395-1407.

ZHANG, Z. F. and QIN, J. (1991). Genetics study on the transparent wing silkworm moth (a primary report). Acta Seric. Sinica 17: 186-187.

Received: May 2004

Reviewed by Referees: July 2004

Modified by Authors and Accepted for Publication: August 2004 